\title{
LORENTZ INVARIANT BARYON CHPT
}

\author{
T. BECHER* \\ Newman Laboratory of Nuclear Studies \\ Cornell University, \\ Ithaca, NY 14853-5001, USA \\ E-mail: becher@mail.lns.cornell.edu
}

\begin{abstract}
Using the example of the elastic $\pi N$-amplitude, we discuss the low energy expansion of QCD amplitudes in the sector with baryon number one. We show that the chiral expansion of these amplitudes breaks down in certain regions of phase space and present a framework which leads to a coherent description throughout the low energy region, while keeping Lorentz and chiral invariance manifest at every stage of the calculation. We explain how to construct a representation of the pion nucleon scattering amplitude in terms of functions of a single variable, which is valid to $O\left(q^{4}\right)$ and properly accounts for the $\pi \pi$ - and $\pi N$-cuts required by unitarity.
\end{abstract}

\section{Introduction}

The chiral symmetry, which the Lagrangian of QCD reveals in the limit of vanishing quark masses, can systematically be incorporated into an effective Lagrangian describing the interaction of the lowest lying baryons and mesons. The effective description is based on a simultaneous expansion of the QCD Greens functions in powers of the light quark masses and the momenta of the Goldstone bosons.

In the vacuum sector of the theory, where the only low energy singularities arise from the propagation of the Goldstone bosons, dimensional regularization yields homogeneous functions of the Goldstone momenta and masses, so that each graph has an unambiguous order in the chiral expansion. The singularity structure in the sector with baryon number one is more complicated. The expansion of the corresponding amplitudes in the momenta and masses of the Goldstone bosons leads to an expansion of the nucleon propagator around the static limit. This expansion of the nucleon propagators is implemented ab initio in the framework called heavy baryon chiral perturbation theory (HBCHPT). However, as we will show in section 2, it fails to converge in part of the low energy region. The breakdown is related to the fact that the expansion of the nucleon propagator ruins in some cases the singularity structure of the amplitudes. This makes it desirable to perform the calculations

* Supported by the Swiss National Science Foundation. The work presented in this article was done in collaboration with H. Leutwyler. 
in a relativistic framework. In doing so, the correct analytic properties of the amplitudes are guaranteed and one can address the question of their chiral expansion in a controlled way.

In the relativistic formulation of the effective theory a technical complication arises from the fact that in a standard regularization prescription, like dimensional regularization, the low energy expansion of the loop graphs starts in general at the same order as the corresponding tree diagrams2. Since the contributions that upset the organization of the perturbation expansion stem from the integration region of large loop momentum of the order of the nucleon mass, they are free of infrared singularities. In section 3 we show that the infrared singular part of the loop integrals can be unambiguously separated from the remainder, whose low energy expansion to any finite order is a polynomial in the momenta and quark masses. Moreover the infrared singular and regular parts of the amplitudes separately obey the Ward identities of chiral symmetry. This ensures that a suitable renormalization of the effective coupling constants removes the infrared regular part altogether, so that we may drop the regular part of the loop integrals and redefine them as the infrared singular part of the integrals in dimensional regularization, a procedure referred to as infrared regularization 3 . The representation of the various quantities of interest obtained in this way combines the virtues of HBCHPT and the relativistic formulation: Both the chiral counting rules and Lorentz invariance and are manifest at every stage of the calculation.

In the meantime this framework has been used to calculate the scalar axials andelectro-magnetic formfactors 9 as well as the elastic pion nucleon amplitudet to fourth order in the chiral expansion. In section 1, we discuss the structure and the low energy expansion of the pion nucleon amplitude. To fourth order in the chiral expansion the amplitude can be given in terms functions of a single variable, either $s, t$ or $u$.

\section{Low energy singularities of one nucleon amplitudes}

The low energy singularities of the one nucleon amplitudes are due to the propagation of pions and nucleons. In the example of the pion nucleon amplitude these include poles at $s=m_{N}^{2}$ and $u=m_{N}^{2}$ due to one nucleon exchange, branch points at $s=\left(M_{\pi}+m_{N}\right)^{2}$ and $u=\left(M_{\pi}+m_{N}\right)^{2}$ due to $\pi N$ intermediate states as well as a branch point at $t=4 M_{\pi}^{2}$ due to $\pi \pi$-intermediate states in the $t$-channel (see figure 1). Note that some of the graphs, like figs. 1 $1 \mathrm{~d}$ and 1] e, also involve a cut for $t>4 m_{N}^{2}$ due to the presence of $\bar{N} N$ intermediate

${ }^{a}$ See also B. Kubis contribution to these proceedings 


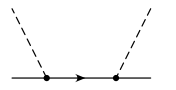

(a)

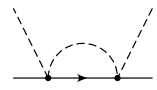

(b)

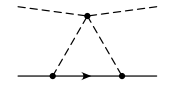

(c)

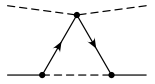

(d)

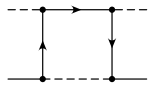

(e)

Figure 1. Examples of contributions to the $\pi N$ scattering amplitude with different singularity structure.

states in the $t$-channel. This singularity lies however outside the low energy region and is absent to any finite order of the chiral expansion.

In performing the low energy expansion we must choose the kinematic variables in such a way that the singularities of the amplitude stay put in order to avoid a breakdown of the expansion near the singularity. We will illustrate this first with the trivial example of the nucleon Born term and then discuss the singularity structure of the triangle graph, figure 1 $1 \mathrm{c}$. In addition to the cut for $t>4 M_{\pi}^{2}$, this graph has a branch point at

$$
t=4 M_{\pi}^{2}-\frac{M_{\pi}^{4}}{m_{N}^{2}}
$$

on the second Riemann sheet. Since the difference between the two singularities is of higher order, they will coalesce if one performs the chiral expansion, causing a breakdown of the expansion in the vicinity of the threshold at $t=4 M_{\pi}^{2}$.

\subsection{Born term}

Denoting the momentum of the incoming nucleon and pion with $P$ resp. $q$, the momentum dependence of the $s$-channel Born contribution to the amplitude, fig. 臬 $\mathrm{a}$ is given by

$$
T_{f i} \propto \frac{\bar{u}^{\prime} \not q\left(\not P+\not q+m_{N}\right) \not q u}{m_{N}^{2}-(P+q)^{2}} .
$$

If we expand in the momentum of the incoming pion, the propagator denominator gets replaced by the geometric series

$$
\frac{1}{m_{N}^{2}-(P+q)^{2}} \rightarrow-\frac{1}{2 P \cdot q}\left\{1-\frac{M_{\pi}^{2}}{2 P \cdot q}+\ldots\right\} .
$$

The location of the singularity has moved to $P \cdot q=0$ or $s=m_{N}^{2}+M_{\pi}^{2}$ and the expansion breaks down for $|P \cdot q|<M_{\pi}^{2} / 2$. Clearly, this problem can be cured by performing the expansion in $q$ at fixed $\tilde{P}=P+q / 2$ instead of 
fixed $P$. Note however that we need to choose appropriate variables for each graph separately in order not to spoil its specific singularity structure. In the example of the graph shown in fig. 11 the expansion at fixed $P$ is convergent, while the expansion at fixed $\tilde{P}$ breaks down in the vicinity of the threshold at $P \cdot q=m_{N} M_{\pi}$.

\subsection{Triangle graph}

The discontinuity of the triangle graph, fig. 1, can be obtained from the extended unitarity relation

$$
\operatorname{Im} f_{ \pm}^{J}(t)=\left\{1-4 M_{\pi}^{2} / t\right\}^{\frac{1}{2}} t_{J}^{I}(t)^{\star} f_{ \pm}^{J}(t), \quad 4 M_{\pi}^{2}<t<16 M_{\pi}^{2} .
$$

The quantities $f_{ \pm}^{J}(t)$ represent the $t$-channel partial waves of the $\pi N$ scattering amplitude. The quantum number $J$ stands for the total angular momentum, while the lower index refers to the spin configuration. There is no need to in addition specify the isospin quantum number, because it is determined by the total angular momentum: $J$ even $\rightarrow I=0, J$ odd $\rightarrow I=1$. The functions $t_{J}^{I}(t)$ denote the partial wave projections of the $\pi \pi$ scattering amplitude. To order $O\left(q^{4}\right)$ the evaluation of this condition is particularly simple, because the $\pi \pi$ scattering amplitude is needed only to leading order, where it is a polynomial. Hence only the partial waves with $J=0,1$ contribute.

To obtain the imaginary part of the triangle graph, we insert the lowest partial waves of the nucleon Born term into the unitarity relation (3). For $J=0$ the pertinent partial waves are

$$
f_{B+}^{0}(t)=\frac{g_{\pi N}^{2} m_{N}}{4 \pi}\left\{f(\kappa)-\frac{t}{4 m^{2}}\right\} \quad \text { and } \quad t_{0}^{0}=\frac{2 t-M_{\pi}^{2}}{32 \pi F_{\pi}^{2}} .
$$

The function $f(\kappa)$ stands for

$$
f(\kappa)=\frac{\arctan \kappa}{\kappa}, \quad \kappa=\frac{\sqrt{\left(t-4 M_{\pi}^{2}\right)\left(4 m_{N}^{2}-t\right)}}{t-2 M_{\pi}^{2}} .
$$

The problem addressed above shows up in this expression: The quantity $\kappa$ represents a term of order $O(1 / q)$ and the standard chiral expansion of $f(\kappa)$ corresponds to an expansion of $\arctan \kappa$ around $\kappa=\infty$. This expansion only converges for $|\kappa|>1$. In the vicinity of the threshold at $t>4 M_{\pi}^{2}$ this condition is not met and the chiral expansion diverges. The breakdown of the expansion is related to the fact that $\arctan \kappa$ has a branch points at $\kappa= \pm i$. To $O\left(q^{4}\right)$, the imaginary part of the invariant amplitude $D^{+}$(see eq. (7) for 
its definition) can be obtained from

$$
\operatorname{Im}_{t} D^{+}=\frac{4 \pi}{m_{N}^{2}} \operatorname{Im} f_{+}^{0}+O\left(q^{5}\right)
$$

The analytic continuation of the scattering amplitude $D^{+}$to the second Riemann sheet therefore contains a branch point at

$$
\frac{\left(t-4 M_{\pi}^{2}\right)\left(4 m_{N}^{2}-t\right)}{\left(t-2 M_{\pi}^{2}\right)^{2}}=-1 \rightarrow t=4 M_{\pi}^{2}-\frac{M_{\pi}^{4}}{m_{N}^{2}} .
$$

\section{Infrared regularization}

Using the example of the self energy integral, we explain how to separate the infrared singular part of the loop integrals from their regular part. As is evident from eqns. (1) and (2), the nucleon propagator counts as a quantity of $O(1 / q)$ at tree level. While the infrared regular part of the loop integrals violates this counting, we show in the following that the infrared singular part respects the counting rules established at tree level.

\subsection{Self-energy integral}

The simplest example of a one loop contribution, the self energy graph, is shown in figure 2 a. The corresponding scalar loop integral has the form

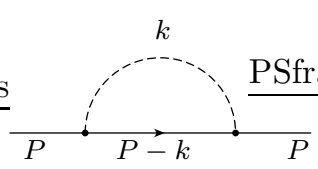

(a)

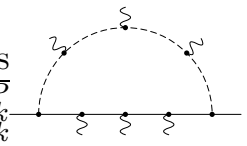

(b)

Figure 2. a.) Self energy graph. b.) Form of the general one loop graph.

$$
H\left(P^{2}\right)=\frac{1}{i} \int \frac{d^{d} k}{(2 \pi)^{d}} \frac{1}{M_{\pi}^{2}-k^{2}} \frac{1}{m_{N}^{2}-(P-k)^{2}}=\frac{1}{i} \int \frac{d^{d} k}{(2 \pi)^{d}} \frac{1}{a} \frac{1}{b} .
$$

We need to analyze the integral for external momenta in the vicinity of the mass shell: $P=m_{N} v+r$, where $v$ is a time-like unit vector and $r$ is a quantity of order $q$. In the limit $M \rightarrow 0$, the integral develops an infrared singularity, generated by small values of the variable of integration, $k=O(q)$. In that region, the first factor in the denominator is of $O\left(q^{2}\right)$, while the second is of order $O(q)$. Accordingly the chiral expansion of the integral contains terms of 
order $q^{d-3}$. The order of the infrared singular part follows from the counting rules at tree level, because it is generated by the region of integration, where the momenta flowing through the propagators are of the same order as in the tree level graphs. The remainder of the integration region does not contain infrared singularities and may thus be expanded in an ordinary Taylor series. An evaluation of the integral at its threshold at $P^{2}=s_{+}=\left(m_{N}+M_{\pi}\right)^{2}$ nicely shows the two parts

$$
H\left(s_{+}\right)=\frac{\Gamma\left(2-\frac{d}{2}\right)}{(4 \pi)^{\frac{d}{2}}(d-3)}\left\{\frac{M_{\pi}^{d-3}}{\left(m_{N}+M_{\pi}\right)}+\frac{m_{N}^{d-3}}{\left(m_{N}+M_{\pi}\right)}\right\}=I+R .
$$

The infrared singular part $I$ is proportional to $M_{\pi}^{d-3}$, while the remainder $R$ is proportional to $m_{N}^{d-3}$ and does therefore not contain a singularity at $M_{\pi}=0$, irrespective of the value of $d$. Since the regular contribution stems from a region where the variable of integration $k$ is of order $O\left(m_{N}\right)$, it violates the tree-level counting rules.

\subsection{Relation to HBCHPT}

The infrared singular part of the loop integrals can be obtained exchanging the order of the chiral expansion and the loop integration. To perform the expansion, both the residual momentum $r_{\mu}=P_{\mu}-m_{N} v_{\mu}$ and the loop momentum $k$ are counted as $O(q)$. Each term in the expansion of the integrand has a trivial dependence on the nucleon mass $m_{N}$ and does therefore not contain a regular part. Performing the loop integration one obtains the infrared part of the integral by summing the individual terms of the series.

Let us illustrate this again for the self energy integral. In this case the expansion amounts to replacing

$$
\frac{1}{m_{N}^{2}-(P-k)^{2}} \rightarrow \frac{1}{2 m_{N} v \cdot(k-r)}+\frac{(k-r)^{2}}{\left\{2 m_{N} v \cdot(k-r)\right\}^{2}}+\ldots
$$

and the relativistic self-energy integral gets replaced by a sum of integrals which arise in HBCHPT (see fig. 3.2):

$$
H \rightarrow I=\sum_{n=1}^{\infty} I_{n}, \quad I_{n}=\int_{k} \frac{1}{M_{\pi}^{2}-k^{2}} \frac{(k-r)^{2(n-1)}}{\left[2 m_{N}(v \cdot k-v \cdot r)\right]^{n}} .
$$

Let us evaluate the integrals at $P^{2}=s_{+}=\left(M_{\pi}+m_{N}\right)^{2}$ by setting $r_{\mu}=M_{\pi} v_{\mu}$. After expressing $(k-r)^{2}=\left(k-M_{\pi} v\right)^{2}$ in terms of the denominators of the integrals and by using the fact that closed loops of static propagators vanish, 


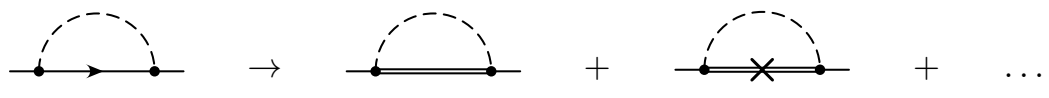

Figure 3. Internal line insertions. The double line denotes the heavy baryon propagator $\left[2 m_{N} v \cdot(k-r)-i \epsilon\right]^{-1}$, the cross an insertion of $(k-r)^{2}$.

we obtain the relation

$$
I_{n}\left(s_{+}\right)=-\frac{M_{\pi}}{m_{N}} I_{n-1}\left(s_{+}\right) .
$$

Since we have expanded the nucleon propagator, the integrals $I_{n}$ depend only trivially on $m_{N}$ (they don't have a regular part) and dimensional analysis gives $I_{1}=c(d) M_{\pi}^{d-3} / m_{N}$. Therefore

$$
I\left(s_{+}\right)=\sum_{n=1}^{\infty} I_{n}\left(s_{+}\right)=\sum_{n=1}^{\infty}(-)^{n} \frac{M_{\pi}^{n}}{m_{N}^{n}} I_{1}\left(s_{+}\right)=c(d) \frac{M_{\pi}^{d-3}}{m_{N}+M_{\pi}} .
$$

An explicit calculation of $I_{1}\left(s_{+}\right)$shows that the constant $c(d)$ is indeed the same as in (4).

In the case of the self-energy integral it is possible to sum the kinematic insertions explicitly $\mathrm{B}$, but for a general integral, the above procedure would be rather clumsy. It however illustrates the relationship between HBCHPT and infrared regularization: The HBCHPT representation of the one nucleon amplitudes is obtained by first performing the chiral expansion and then the loop integration. The infrared regularized amplitudes can be obtained from HBCHPT by resumming the graphs with kinematic insertions.

\subsection{Feynman parameterization}

The explicit expression for the loop integral $H\left(P^{2}\right)$ in $d$ dimensions is lengthy, but the splitting into the infrared singular part $I$ and regular part $R$ is easily obtained in the Schwinger-Feynman representation of the integral.

$$
\begin{aligned}
H & =\int \frac{d^{d} k}{(2 \pi)^{d}} \frac{1}{a} \frac{1}{b}=\int_{0}^{1} d z \int \frac{d^{d} k}{(2 \pi)^{d}} \frac{1}{[(1-z) a+z b]^{2}} \\
& =\int_{0}^{\infty}-\int_{1}^{\infty} d z \int \frac{d^{d} k}{(2 \pi)^{d}} \frac{1}{[(1-z) a+z b]^{2}}=I+R .
\end{aligned}
$$

The case of a general one loop integral (see fig.2 $2 \mathrm{~b}$ ) can be reduced to the self-energy integral, by first using the ordinary Feynman parameterization to 
combine all the meson propagators into a single one. After combining also the nucleon propagators in the same way, the general integral can be written as an integral over the self-energy integral $H$ and derivatives with respect to its masses. Its infrared part is then obtained by replacing $H$ by the Feynman parameterization for $I$ given in (6). Feynman parameterizations of the infrared singular apd regular parts of a general one loop integral can be found in the literature 3 .

\subsection{Chiral symmetry}

In the framework of the relativistic effective theory, the evaluation of an amplitude to one loop yields three contributions, arising from (a) the tree graphs, (b) the infrared singular and (c) the infrared regular part of the one loop graphs. At a given order in the chiral expansion, the one particle irreducible components of the regular part are polynomial in the external momenta. In coordinate space this contribution is given by local terms and thus equivalent to the tree graphs generated by a suitable Lagrangian $\Delta \mathcal{L}$. So that, if we replace the effective Lagrangian by

$$
\mathcal{L}_{\text {eff }}^{\prime}=\mathcal{L}_{\text {eff }}+\Delta \mathcal{L}
$$

we may drop the regular part of the loop integrals.

We show now that the modified Lagrangian $\mathcal{L}_{\text {eff }}^{\prime}$ occurring in our framework respects the Ward identities of chiral symmetry. In the limit of vanishing quark masses these identities interrelate the Green's functions of the form

$$
<N\left(P^{\prime}, s^{\prime}\right)\left|\mathbf{T}\left\{O_{1}\left(x_{1}\right) \ldots O_{n}\left(x_{n}\right)\right\}\right| N(P, s)>,
$$

where the operators $O_{i}$ stand for vector, axial-vector, scalar and pseudo-scalar quark-currents.

Since $\mathcal{L}_{\text {eff }}$ is chirally symmetric, the tree level amplitudes (a) respect the Ward identities. Because dimensional regularization preserves the symmetry of $\mathcal{L}_{\text {eff }}$, the same is true for the loop contribution (b)+(c) for arbitrary values of the regularization parameter $d$. Since (b) involves only fractional and (c) only integer powers of the chiral expansion parameter, the sum of the two can only fulfill the Ward identities if they are separately fulfilled by the two parts and we conclude that the Lagrangian $\Delta \mathcal{L}$, which generates the contributions (c) is indeed chirally symmetric.

\section{Representation of the $\pi N$-scattering amplitude at $O\left(q^{4}\right)$}

The virtues of CHPT are twofold: One one hand chiral symmetry interrelates different amplitudes, on the other hand the framework allows one to obtain a 


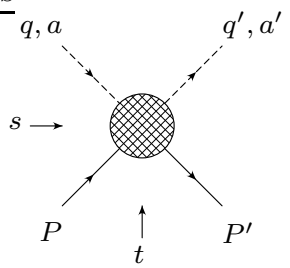

Figure 4. Kinematics of the elastic $\pi N$ scattering amplitude $P, q\left(P^{\prime}, q^{\prime}\right)$ denote the momentum of the incoming (outgoing) nucleons and pions, and $a\left(a^{\prime}\right)$ stands for the isospin indices of the incoming (outgoing) pion.

parameterization of a given amplitude in a number of low energy parameters not fixed by the symmetry.

In the following we focus on the energy dependence of the elastic pion nucleon scattering amplitude in the isospin limit, obtained on the basis of a calculation in infrared regularization to $O\left(q^{4}\right)$. We explain, how one arrives at a representation of the amplitude in terms of functions of a single variable without invoking an expansion of its infrared singularities. As discussed in section 2, the chiral expansion of the triangle graph does not converge around its threshold. The expansion of the other graphs is convergent, but the convergence is in general rather slow and depends on the choice of the kinematic variables, which are kept fixed while one performs the expansion.

In section 2, we have listed some of the analytic properties of the scattering amplitude. In the chiral representation of the amplitude, the corresponding cuts are described by loop integrals. These loop integrals in general also contain singularities outside the low energy region and we will in the following simplify the representation of the amplitude by chirally expanding the contributions from these singularities. As a result of these simplifications, we can represent the amplitude as the sum of the Born term, a polynomial containing the low energy constants and nine functions of a single variable, which are given in terms of integrals over the imaginary parts of the loop integrals.

In the context of our low energy analysis, the standard decomposition of the scattering amplitude into invariant amplitudes $A$ and $B$ is not well suited, because their leading order contributions cancel. Replacing $A$ by $D=A+\nu B$, with $\nu=(s-u) / 4 m_{N}$, the scattering amplitude is given by

$$
T^{ \pm}=\bar{u}^{\prime}\left\{D^{ \pm}-\frac{1}{4 m_{N}}\left[\phi^{\prime}, \not q\right] B^{ \pm}\right\} u
$$


where the upper index refers to the isospin decomposition

$$
T_{a^{\prime} a}=\delta_{a^{\prime} a} T^{+}+\frac{1}{2}\left[\tau_{a^{\prime}}, \tau_{a}\right] T^{-} .
$$

The evaluation of the chiral perturbation series to one loop allows us to calculate the amplitudes $D^{ \pm}$and $B^{ \pm}$to $O\left(q^{4}\right)$ and $O\left(q^{2}\right)$, respectively.

\subsection{Simplification of the $t$-dependence of the amplitude}

The triangle graph with two nucleon propagators, fig. 11d, and the box graph, fig. 11e, have a cut for $t>4 m_{N}^{2}$. Because this cut lies outside the low energy region, we can expand the corresponding graphs in the variable $t$. The higher order contributions in $t$ are suppressed as $t / 4 m_{N}^{2}$ and are therefore also of higher order in the chiral expansion. After this simplification, the amplitude can be expressed in terms of functions of a single variable: The contribution of the graphs with an $s$-channel cut is a linear function of $t$ and the contribution of the graphs with a $t$-channel cut is linear in $\nu$.

\subsection{Dispersive representation}

By construction, the infrared regular part of the loop integrals has no singularities in the low energy region. It however has unphysical singularities outside the low energy region ${ }^{3}$ and since the infrared part is given by the difference between the full integrals and their regular part, these singularities are also present in the infrared part. The infrared part of the self energy integral, for example, has a cut for $s<0$ and a pole at $s=0$. Also these singularities can safely be chirally expanded. Let us illustrate in the example of the forward amplitude $\bar{D}^{+}(\nu, t=0)$ how this can be achieved (we use the bar to indicate that the pseudo-vector Born term has been subtracted from $D^{+}$). In a first step, we expand the amplitude around $\nu=0$ to $O\left(\nu^{4}\right)$ :

$$
\bar{D}^{+}(\nu, 0)=d_{0}+d_{1} \nu^{2}+d_{2} \nu^{4}+O\left(\nu^{6}\right)
$$

The absence of odd powers of $\nu$ is due to crossing symmetry. Note that we have not expanded the coefficients $d_{i}$ in powers of the quark masses, because this expansion converges only slowly, especially in the case of $d_{2}$. Once its contributions to the polynomial part are subtracted, the remaining contribution from the physical $s$ - and $u$-channel cut is given by the dispersion integral

$$
\hat{D}^{+}(\nu)=\frac{2}{\pi} \int_{M_{\pi}}^{\infty} \frac{\mathrm{d} \nu^{\prime}}{\nu^{\prime 2}-\nu^{2}} \frac{\nu^{6}}{\nu^{\prime 5}} \operatorname{Im}_{s} D^{+}(\nu, 0) .
$$


$\operatorname{Im}_{s} D^{+}(\nu, 0)$ denotes the discontinuity over the right hand cut of the $s$-channel graphs, which is a consequence of unitarity and is the same in dimensional and infrared regularization.

If we subtract from the amplitude both the polynomial and the dispersion integral, we are left with

$$
D_{\text {reg }}^{+}(\nu)=\bar{D}^{+}(\nu, 0)-\hat{D}^{+}(\nu)-d_{0}-d_{1} \nu^{2}-d_{2} \nu^{4},
$$

which receives contributions only from the singularities outside the low energy region. Since we have accounted for all infrared singularities and since $D_{\text {reg }}^{+}(\nu, 0)$ is proportional to $\nu^{6}$, its chiral expansion involves only terms of order $O\left(q^{6}\right)$ and higher and we can drop $D_{\text {reg }}^{+}$to the accuracy of our calculation. The representation of the forward amplitude now takes the form

$$
\bar{D}^{+}(\nu, 0)=d_{0}+d_{1} \nu^{2}+d_{2} \nu^{4}+\hat{D}^{+}(\nu)+O\left(p^{4}\right) .
$$

The coefficients $d_{i}$ contain low energy constants, whose values are not fixed by the symmetry. By construction $\hat{D}^{+}(\nu)$ does not contribute to the coefficients $d_{i}$, so that we can identify these constants with the lowest coefficients of the subthreshold-expansion of Höhler and collaborators

We can follow the same strategy to obtain a dispersive representation of $D^{+}$for nonzero values of $t$. This representation involves three more subtraction constants, corresponding to the coefficients of $t, \nu^{2} t$ and $t^{2}$ of the subthreshold expansion, as well as a dispersion integral over the $t$-channel cut. After the simplifications made in section 4.1, the $s$-channel imaginary part is linear in $t$ and one needs an additional dispersion integral, which supplies the contributions of the $s$ - and $u$-channel cut which are proportional to $t$. In the case of the amplitudes $B^{ \pm}$, the $s$-channel imaginary part is independent of $t$, so that one function suffices to describe the $s$-channel cut contribution for each of these. Furthermore the $t$-channel imaginary part of $B^{+}$vanishes to the order of our calculation, so that we need a total of nine functions of a single variable to account for all the low energy singularities of the amplitude to $O\left(q^{4}\right)$.

\section{Conclusion}

We have presented a formulation for BCHPT (infrared regularization) in which both, Lorentz invariance and the chiral power counting rules are preserved at every stage of the calculation. The method relies on the fact that for noninteger values of the regularization parameter $d$, the infrared singular part of the relativistic loop integrals can be unambiguously separated from the remainder. Since the remainder is free of infrared singularities, it is a 
polynomial in the external momenta and quark masses to any finite order of the chiral expansion and can be absorbed into a redefinition of the coupling constants of the effective Lagrangian.

We have shown that the chiral expansion of the $\pi N$-scattering amplitude does not converge in the vicinity of the threshold at $t=4 M_{\pi}^{2}$. The breakdown of the expansion is related to the fact that the expansion of the nucleon propagator around the static limit destroys some of the analytic properties of the amplitude. In the relativistic formulation of the effective theory the correct analytic structure is guaranteed and one obtains a representation, which is valid in the entire low energy region.

The representation of a given amplitude obtained in the relativistic framework contains in general also singularities outside the low energy region. We have shown how to simplify the representation of the $\pi N$-scattering amplitude at $O\left(q^{4}\right)$, by chirally expanding the contributions of these singularities. As a result of these simplifications, the scattering amplitude can be written as the Born term, a polynomial containing the low energy constants and nine functions of a single variable. The corresponding functions are given in terms of dispersion integrals over the imaginary parts of the one loop graphs.

\section{References}

1. E. Jenkins and A. V. Manohar, Phys. Lett. B 255, 558 (1991). V. Bernard, N. Kaiser, J. Kambor and U.-G. Meissner, Nucl. Phys. B 388, 315 (1992).

2. J. Gasser, M. E. Sainio and A. Švarc, Nucl. Phys. B 307, 779 (1988).

3. T. Becher and H. Leutwyler, Eur. Phys. J. C 9, 643 (1999)

4. H.-B. Tang, A new approach to chiral perturbation theory for matter fields, hep-ph/9607436

P. J. Ellis and H.-B. Tang, Phys. Rev. C 57, 3356 (1998)

5. J. Schweizer, Low energy representation for the axial form factor of the nucleon, diploma thesis, Bern 2000.

6. B. Kubis and U. Meissner, Low energy analysis of the nucleon electromagnetic form factors, hep-ph/0007056.

B. Kubis and U. Meissner, Baryon form factors in chiral perturbation theory, hep-ph/0010283.

7. T. Becher and H. Leutwyler, to appear.

8. G. Höhler, in Landolt-Börnstein, 9b2, ed. H. Schopper (Springer, Berlin, 1983). 